\title{
Qualitative Study of a 4D Chaos Financial System
}

\author{
Fuchen Zhang $\mathbb{D}^{1,2}$ Gaoxiang Yang $\mathbb{D}^{3},{ }^{3}$ Yong Zhang, ${ }^{4}$ Xiaofeng Liao, ${ }^{5}$ and Guangyun Zhang ${ }^{1}$ \\ ${ }^{1}$ College of Mathematics and Statistics, Chongqing Technology and Business University, Chongqing 400067, China \\ ${ }^{2}$ Mathematical Postdoctoral Station, College of Mathematics and Statistics, Southwest University, Chongqing 400716, China \\ ${ }^{3}$ School of Mathematics and Statistics, Ankang University, Shaanxi 725000, China \\ ${ }^{4}$ College of Mathematics and Statistics, Henan Polytechnic Institute, Nanyang 473000, China \\ ${ }^{5}$ College of Electronic and Information Engineering, Southwest University, Chongqing 400716, China
}

Correspondence should be addressed to Fuchen Zhang; zhangfuchen1983@163.com

Received 11 February 2018; Accepted 17 May 2018; Published 16 July 2018

Academic Editor: Roberto Natella

Copyright ( 2018 Fuchen Zhang et al. This is an open access article distributed under the Creative Commons Attribution License, which permits unrestricted use, distribution, and reproduction in any medium, provided the original work is properly cited.

\begin{abstract}
Some dynamics of a new $4 \mathrm{D}$ chaotic system describing the dynamical behavior of the finance are considered. Ultimate boundedness and global attraction domain are obtained according to Lyapunov stability theory. These results are useful in estimating the Lyapunov dimension of attractors, Hausdorff dimension of attractors, chaos control, and chaos synchronization. We will also present some simulation results. Furthermore, the volumes of the ultimate bound set and the global exponential attractive set are obtained.
\end{abstract}

\section{Introduction}

Chaotic systems are characterized by their extreme sensitivity both to initial conditions and to system parameters. The study of various aspects of chaotic systems has received great interest among scientists from various fields due to their numerous potential applications in science and engineering [1-30]. Among such aspects, the problem of investigating the ultimate boundedness of chaotic systems and hyperchaotic systems is an important subject. Since the discovery of the famous Lorenz chaotic attractor [1], ultimate boundedness of the Lorenz attractor has been investigated by Leonov et al. in a series of articles [7, 10]. Since then many papers have studied ultimate boundedness of other chaotic systems [25-32]. However, the approach taken in each is only suitable for that particular chaotic system. It is very difficult to propose a universal approach to estimate the bounds for an arbitrary chaotic system. Zhang et al. studied the ultimate boundedness of the Lü system and the Chen system by using the Lyapunov stability theory and optimization method [25-27]. Particularly, Liao et al. studied the ultimate boundedness of the Yang-Chen system by using geometric and algebraic methods [31]. Zhang et al. studied the ultimate boundedness of a novel finance chaotic system by using the
Lyapunov stability theory and iterative method [32]. To this end, it is necessary to study the bounds of the new chaotic systems.

Recently, a financial chaos dynamical system is reported as follows $[32,33]$ :

$$
\begin{aligned}
& \frac{d x}{d t}=z+(y-a) x, \\
& \frac{d y}{d t}=1-b y-x^{2}, \\
& \frac{d z}{d t}=-x-c z .
\end{aligned}
$$

System (1) models a financial dynamical system composed of product, money, bond, and labor force. The variables $x, y$, and $z$ denote the interest rate, the investment demand, and the price index, respectively. The positive parameters $a, b$, and $c$ denote the saving amount, the per investment cost, and the demand elasticity of commercials, respectively. The factors that induce the change of the interest rate $x$ mainly come from two aspects: the price index and the surplus between the investment demand and the savings amount. The changing rate of $y$ is determined by the benefit rate of investment (we assume that the rate is constant during 
a certain period), the feedback of the investment demand, and the interest rate. The change of the price index $z$ is controlled by the real interest rate and price index.

According to the financial dynamical system (1), by adding a variable $u$, one gets a new $4 \mathrm{D}$ financial chaos dynamical system as follows:

$$
\begin{aligned}
& \frac{d x}{d t}=z+(y-a) x, \\
& \frac{d y}{d t}=1-b y-x^{2}, \\
& \frac{d z}{d t}=-x-c z+d u, \\
& \frac{d u}{d t}=-k u-m z .
\end{aligned}
$$

where the variable $u$ denotes control input and economically state intervention to balance the economic environment and $a, b, c, d, k, m$ are positive real parameters of system (2).

The Lyapunov exponents of the financial system (2) are calculated numerically for the parameter values $a=0.9$, $b=0.2, c=1.5, d=1, k=0.05$, and $m=0.005$ with the initial state $\left(x_{0}, y_{0}, z_{0}, u_{0}\right)=(0,1, \ldots, 0.5,0)$. System (2) has the Lyapunov exponents as $\lambda_{\mathrm{LE}_{1}}=0.1740, \lambda_{\mathrm{LE}_{2}}=0.1314, \lambda_{\mathrm{LE}_{3}}=$ 0.0000 , and $\lambda_{\mathrm{LE}_{4}}=-15.6059$ for the above parameters (see $[8,9]$ for a detailed discussion of Lyapunov exponents of strange attractors in chaos dynamical systems).

The chaotic attractor of system (2) in $x O y z$ space for the positive parameters $a=0.9, b=0.2, c=1.5, d=1, k=0.05$, and $m=0.005$ is shown in Figure 1 .

Remark 1. The chaotic attractor is one of the important concepts of dynamical systems. Although system (2) has a chaotic attractor for the positive parameters $a=0.9, b=0.2$, $c=1.5, d=1, k=0.05$, and $m=0.005$, the type of the chaotic attractor of system (2) is still unknown. It is interesting to discuss whether the chaotic attractor of system (2) is hidden or self-excited in the future (see the excellent papers $[17,19]$ for a detailed discussion of the chaotic attractor of dynamical systems).

This paper is organized as follows. In the next section, we will study the bounds for the chaotic attractors (2) using the Lyapunov stability theory and optimization method. To validate the ultimate bound estimation, numerical simulations are also provided. Finally, we will give some concluding remarks in Section 3.

\section{Main Properties}

Theorem 1. Suppose that $a>0, b>0, c>0, k>0, d \neq 0, m>0$. Let $X(t)=(x(t), y(t), z(t), u(t))$ be an arbitrary solution of system (2). Then, the following set

$$
\Omega=\left\{(x, y, z, u) \mid m x^{2}+m y^{2}+m z^{2}+d u^{2} \leq R^{2}\right\},
$$

is the ultimate bound set and positively invariant set of system (2), where

$$
R^{2}= \begin{cases}\frac{m}{4 a(b-a)}, & c \geq a, k \geq a, b \geq 2 a, \\ \frac{m}{4 c(b-c)}, & a>c, k>c, b \geq 2 c, \\ \frac{m}{4 k(b-k)}, & a>k, c>k, b \geq 2 k, \\ \frac{m}{b^{2}}, & b<2 a, b<2 c, b<2 k .\end{cases}
$$

Proof 1. Define the Lyapunov-like function

$$
V(X)=m x^{2}+m y^{2}+m z^{2}+d u^{2},
$$

where $X(t)=(x(t), y(t), z(t), u(t))$. Computing the derivative of $V(X)$ along the trajectory of (2), we have

$$
\begin{aligned}
\left.\frac{d V(X)}{d t}\right|_{(2)}= & 2 m x \frac{d x}{d t}+2 m y \frac{d y}{d t}+2 m z \frac{d z}{d t}+2 d u \frac{d u}{d t} \\
= & 2 m x(z+x y-a x)+2 m y\left(1-b y-x^{2}\right) \\
& +2 m z(-x-c z+d u)+2 d u(-k u-m z) \\
= & -2 a m x^{2}-2 b m y^{2}+2 m y-2 c m z^{2}-2 d k u^{2} \\
= & -2 a m x^{2}-2 b m\left(y-\frac{1}{2 b}\right)^{2}-2 c m z^{2}-2 d k u^{2}+\frac{m}{2 b} .
\end{aligned}
$$

Obviously, the surface $\Gamma_{1}$, that is, defined by

$$
\Gamma_{1}=\left\{X \mid a m x^{2}+b m\left(y-\frac{1}{2 b}\right)^{2}+c m z^{2}+d k u^{2}=\frac{m}{4 b}\right\},
$$

is an ellipsoid in $R^{4} \forall a>0, b>0, c>0, k>0, d \neq 0$, and $m>0$. Outside $\Gamma_{1}:(d V(X) / d t)<0$, while inside $\Gamma_{1}:(d V(X) / d t)>0$. Thus, the maximum value of $V(X)$ can only be reached on $\Gamma_{1}$. Since the $V(X)$ is a continuous function and $\Gamma_{1}$ is a bounded closed set, then the function (5) can reach its maximum value $\max _{X \in \Gamma_{1}} V(X)=R^{2}$ on the surface $\Gamma_{1}$ defined in (7).

Obviously, $\quad\left\{X \mid V(X) \leq \max _{X \in \Gamma_{1}} V(X)=R^{2}\right\}$ contains the solutions of system (2). By solving the following conditional extremum problem, one can get the maximum value of the function (5) as follows:

$$
\begin{array}{ll}
\max & V(X)=\max \left\{m x^{2}+m y^{2}+m z^{2}+d u^{2}\right\}, \\
\text { s.t. } & a m x^{2}+b m\left(y-\frac{1}{2 b}\right)^{2}+c m z^{2}+d k u^{2}=\frac{m}{4 b} .
\end{array}
$$

That is to say,

$$
\begin{array}{ll}
\max & V(X)=\max \left\{m x^{2}+m y^{2}+m z^{2}+d u^{2}\right\}, \\
\text { s.t. } & \frac{m x^{2}}{m / 4 b a}+\frac{m(y-(1 / 2 b))^{2}}{m / 4 b^{2}}+\frac{m z^{2}}{m / 4 b c}+\frac{d u^{2}}{m / 4 b k}=1 .
\end{array}
$$




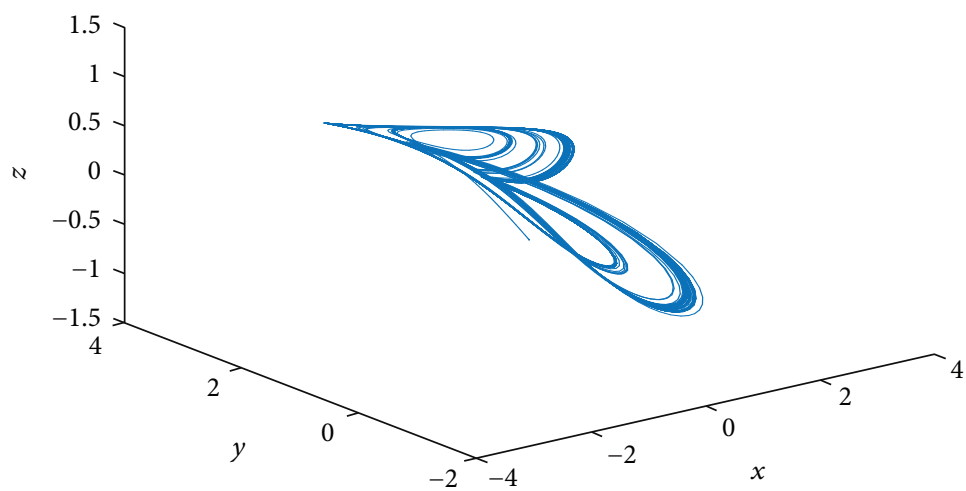

FIGURE 1: The chaotic attractor of system (2) in $x O y z$ space with $a=0.9, b=0.2, c=1.5, d=1, k=0.05, m=0.005$, and $\left(x_{0}, y_{0}, z_{0}, u_{0}\right)=$ $(0,1, \ldots, 0.5,0)$.

Let us take $\sqrt{m} x=x_{1}, \sqrt{m} y=y_{1}, \sqrt{m} z=z_{1}, \sqrt{d} u=u_{1}$ as the new variables, then (9) transforms into the following form:

$$
\begin{array}{ll}
\max & V(X)=\max \left\{x_{1}^{2}+y_{1}^{2}+z_{1}^{2}+u_{1}^{2}\right\} \\
\text { s.t. } & \frac{x_{1}^{2}}{m / 4 b a}+\frac{\left(y_{1}-(\sqrt{m} / 2 b)\right)^{2}}{m / 4 b^{2}}+\frac{z_{1}^{2}}{m / 4 b c}+\frac{u_{1}^{2}}{m / 4 b k}=1 .
\end{array}
$$

According to the Lagrange multiplier method, we can easily get the above conditional extremum problem (10) as

$$
R^{2}= \begin{cases}\frac{m}{4 a(b-a)}, & c \geq a, k \geq a, b \geq 2 a, \\ \frac{m}{4 c(b-c)}, & a>c, k>c, b \geq 2 c, \\ \frac{m}{4 k(b-k)}, & a>k, c>k, b \geq 2 k, \\ \frac{m}{b^{2}}, & b<2 a, b<2 c, b<2 k .\end{cases}
$$

Finally, it is easy to show that (3) is the ultimate bound set and positively invariant set of system (2).

This completes the proof.

\section{Remark 2.}

(i) When $a=0.9, b=0.2, c=1.5, d=1, k=0.05$, and $m=0.005$, then we can obtain that

$$
\begin{aligned}
\Omega_{1}=\{ & (x, y, z, u) \mid 0.005 x^{2}+0.005 y^{2}+0.005 z^{2} \\
& \left.+u^{2} \leq 0.41^{2}\right\},
\end{aligned}
$$

which is the ultimate bound set and positively invariant set of system (2) according to Theorem 1. Figure 2 shows the chaotic attractor of system (2) in the $x O y z$ space defined by $\Omega_{1}$.

(ii) We can figure out that the volume of the set $\Omega$ in (3) is $v(\Omega)=\pi^{2} R^{4} / 2 m \sqrt{m d}$ according to Theorem 1 .

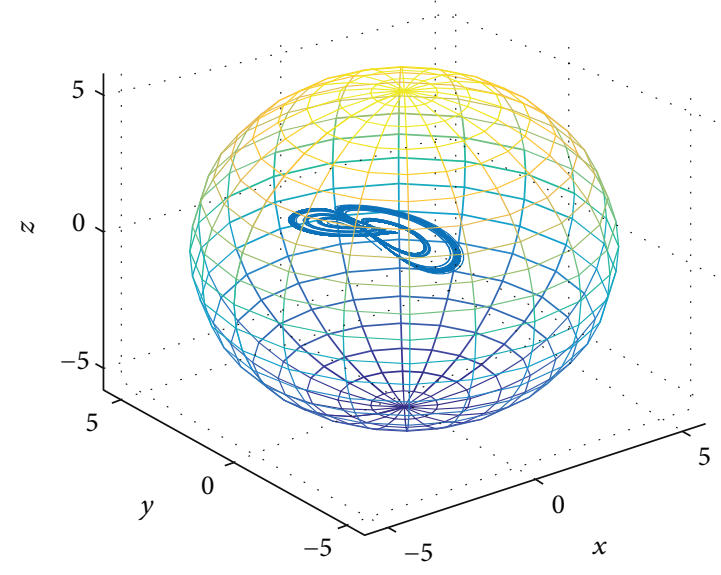

Figure 2: The chaotic attractor of system (2) in the $x O y z$ space defined by $\Omega_{1}$ with $a=0.9, b=0.2, c=1.5, d=1, k=0.05$, and $m=0.005$.

Though Theorem 1 gives the ultimate bound set and positively invariant set of the financial chaos system (2), it does not give the global exponential attractive set of system (2). The global exponential attractive set of system (2) is described by the following Theorem 2 .

Theorem 2. Suppose that $a>0, b>0, c>0, k>0, d \neq 0$, and $m>0$, and let

$$
\begin{aligned}
V(X) & =m x^{2}+m y^{2}+m z^{2}+d u^{2}, \\
L^{2} & =\frac{m}{\theta b}, \\
\theta & =\min (a, b, c, k)>0 .
\end{aligned}
$$

Then, the estimation,

$$
\left[V(X(t))-L^{2}\right] \leq\left[V\left(X\left(t_{0}\right)\right)-L^{2}\right] e^{-\theta\left(t-t_{0}\right)},
$$

holds for system (2), and thus

$$
\Psi=\left\{X \mid V(X) \leq L^{2}\right\},
$$

which is the globally exponential attractive set of system (2), that is, $\varlimsup_{t \rightarrow+\infty} V(X(t)) \leq L^{2}$. 
Proof 2. Define the following function of a variable

$$
f(y)=-b m y^{2}+2 m y,
$$

then we can get

$$
\max _{y \in R} f(y)=\frac{m}{b}
$$

Define the Lyapunov function

$$
V(X)=m x^{2}+m y^{2}+m z^{2}+d u^{2} .
$$

Differentiating the Lyapunov function $V(X)$ in (18) with respect to time $t$ along the trajectory of system (2), when $V(X(t))>L^{2}$ and $V\left(X\left(t_{0}\right)\right)>L^{2}$, we have

$$
\begin{aligned}
\frac{\left.d V(X)\right|_{(2)}=}{d t} \mid & m x \frac{d x}{d t}+2 m y \frac{d y}{d t}+2 m z \frac{d z}{d t}+2 d u \frac{d u}{d t} \\
= & 2 m x(z+x y-a x)+2 m y\left(1-b y-x^{2}\right) \\
& +2 m z(-x-c z+d u)+2 d u(-k u-m z) \\
= & -2 a m x^{2}-2 b m y^{2}+2 m y-2 c m z^{2}-2 d k u^{2} \\
\leq & -a m x^{2}-b m y^{2}-c m z^{2}-d k u^{2}-b m y^{2}+2 m y \\
\leq & -a m x^{2}-b m y^{2}-c m z^{2}-d k u^{2}+f(y) \\
\leq & -a m x^{2}-b m y^{2}-c m z^{2}-d k u^{2}+\max _{y \in R} f(y) \\
\leq & -a m x^{2}-b m y^{2}-c m z^{2}-d k u^{2}+\frac{m}{b} \\
\leq & -\theta V(X)+\frac{m}{b}=-\theta\left[V(X)-\frac{m}{b \theta}\right] \\
= & -\theta\left[V(X)-L^{2}\right]<0 .
\end{aligned}
$$

Thus, we have

$$
\left[V(X(t))-L^{2}\right] \leq\left[V\left(X\left(t_{0}\right)\right)-L^{2}\right] e^{-\theta\left(t-t_{0}\right)},
$$

and

$$
\varlimsup_{t \rightarrow+\infty} V(X(t)) \leq L^{2}
$$

which clearly shows that $\Psi=\left\{X \mid V(X) \leq L^{2}\right\}$ is the globally exponential attractive set of system (2).

The proof is complete.

Remark 3.

(i) We can figure out that the volume of the set $\Psi$ in (15) is $v(\Psi)=\pi^{2} L^{4} / 2 m \sqrt{m d}$ according to Theorem 2 .

\section{Conclusions}

In this paper, we considered some dynamics of a new $4 \mathrm{D}$ financial chaos system. We obtained the ultimate boundedness and global exponential attractive sets of this system according to Lyapunov stability theory. MATLAB simulations show that the proposed method is effective. Characteristic time scales of the $4 \mathrm{D}$ financial system, the homoclinic orbits, and heteroclinic orbits of the 4D financial system will be taken into consideration in the future.

\section{Data Availability}

No data were used to support this study.

\section{Conflicts of Interest}

The authors declare that they have no conflicts of interest.

\section{Authors' Contributions}

All authors have read and approved the final manuscript.

\section{Acknowledgments}

This paper is supported by National Natural Science Foundation of China (Grant nos. 11501064 and 11426047), the Scientific and Technological Research Program of Chongqing Municipal Education Commission (Grant no. KJ1500605), the Research Fund of Chongqing Technology and Business University (Grant nos. 2014-56-11, KFJJ2017066, and 2018106), China Postdoctoral Science Foundation (Grant no. 2016M590850), Chongqing Postdoctoral Science Foundation Special Funded Project (Grant no. Xm2017174), 2016 Chongqing Social Science Planning Major Entrustment Project "Frontier theory research on census quality assessment" (Grant no. 2016WT03), the National Key Research and Development Program of China (Grant no. 2016YFB0800601), in part by the National Natural Science Foundation of China (Grant no. 61472331), and Natural Science Foundation of Shaanxi Provincial Education Department (Grant no. 15JK1016). The authors thank Professors Jinhu Lü in Chinese Academy of Sciences, Lennart Stenflo in the Royal Swedish Academy of Sciences, Gennady A. Leonov in the Saint-Petersburg State University, and Min Xiao in Nanjing University of Posts and Telecommunications for their assistance.

\section{References}

[1] E. N. Lorenz, "Deterministic nonperiodic flow," Journal of the Atmospheric Sciences, vol. 20, no. 2, pp. 130-141, 1963.

[2] O. Rössler, "An equation for hyperchaos," Physics Letters A, vol. 71, no. 2-3, pp. 155-157, 1979.

[3] L. Chua, M. Komura, and T. Matsumoto, "The double scroll family," IEEE Transactions on Circuits and Systems, vol. 33, no. 11, pp. 1072-1118, 1986.

[4] G. Chen and T. Ueta, "Yet another chaotic attractor," International Journal of Bifurcation and Chaos, vol. 9, no. 7, pp. 1465-1466, 1999.

[5] J. Lü and G. Chen, "A new chaotic attractor coined," International Journal of Bifurcation and Chaos, vol. 12, no. 3, pp. 659-661, 2002.

[6] G. A. Leonov and N. V. Kuznetsov, "On differences and similarities in the analysis of Lorenz, Chen, and $\mathrm{Lu}$ 
systems," Applied Mathematics and Computation, vol. 256, pp. 334-343, 2015.

[7] G. A. Leonov, "Bounds for attractors and the existence of homoclinic orbits in the Lorenz system," Journal of Applied Mathematics and Mechanics, vol. 65, no. 1, pp. 19-32, 2001.

[8] P. Frederickson, J. L. Kaplan, E. D. Yorke, and J. A. Yorke, "The Liapunov dimension of strange attractors," Journal of Differential Equations, vol. 49, no. 2, pp. 185-207, 1983.

[9] A. Wolf, J. B. Swift, H. L. Swinney, and J. A. Vastano, "Determining Lyapunov exponents from a time series," Physica D, vol. 16, no. 3, pp. 285-317, 1985.

[10] G. A. Leonov, A. Bunin, and N. Koksch, "Attractor localization of the Lorenz system," ZAMM - Journal of Applied Mathematics and Mechanics/Zeitschrift für Angewandte Mathematik und Mechanik, vol. 67, no. 12, pp. 649-656, 1987.

[11] X. X. Liao, Y. L. Fu, and S. L. Xie, "On the new results of global attractive set and positive invariant set of the Lorenz chaotic system and the applications to chaos control and synchronization," Science in China Series F: Information Sciences, vol. 48, no. 3, pp. 304-321, 2005.

[12] G. A. Leonov, "Generalized Lorenz equations for acousticgravity waves in the atmosphere. Attractors dimension, convergence and homoclinic trajectories," Communications on Pure and Applied Analysis, vol. 16, no. 6, pp. 2253-2267, 2017.

[13] X. Y. Wang and M. J. Wang, "A hyperchaos generated from Lorenz system," Physica A, vol. 387, no. 14, pp. 3751-3758, 2008.

[14] G. A. Leonov, "General existence conditions of homoclinic trajectories in dissipative systems. Lorenz, Shimizu-Morioka, Lu and Chen systems," Physics Letters A, vol. 376, no. 45, pp. 3045-3050, 2012.

[15] F. C. Zhang, Y. L. Shu, and H. L. Yang, "Bounds for a new chaotic system and its application in chaos synchronization," Communications in Nonlinear Science and Numerical Simulation, vol. 16, no. 3, pp. 1501-1508, 2011.

[16] X. Y. Wang and M. J. Wang, "Dynamic analysis of the fractional-order Liu system and its synchronization," Chaos, vol. 17, no. 3, article 033106, 2007.

[17] G. A. Leonov, N. V. Kuznetsov, and T. N. Mokaev, "Homoclinic orbits, and self-excited and hidden attractors in a Lorenz-like system describing convective fluid motion," The European Physical Journal Special Topics, vol. 224, no. 8, pp. 1421-1458, 2015.

[18] N. V. Kuznetsov, T. N. Mokaev, and P. A. Vasilyev, "Numerical justification of Leonov conjecture on Lyapunov dimension of Rossler attractor," Communications in Nonlinear Science and Numerical Simulation, vol. 19, no. 4, pp. 1027-1034, 2014.

[19] G. A. Leonov and N. V. Kuznetsov, "Hidden attractors in dynamical systems. From hidden oscillations in HilbertKolmogorov, Aizerman, and Kalman problems to hidden chaotic attractor in Chua circuits," International Journal of Bifurcation and Chaos, vol. 23, no. 01, article 1330002, 2013.

[20] G. A. Leonov, N. V. Kuznetsov, M. A. Kiseleva, E. P. Solovyeva, and A. M. Zaretskiy, "Hidden oscillations in mathematical model of drilling system actuated by induction motor with a wound rotor," Nonlinear Dynamics, vol. 77, no. 1-2, pp. 277288, 2014.

[21] H. J. Liu, X. Y. Wang, and Q. L. Zhu, "Asynchronous anti-noise hyper chaotic secure communication system based on dynamic delay and state variables switching," Physics Letters A, vol. 375, no. 30-31, pp. 2828-2835, 2011.

[22] Y. Q. Zhang and X. Y. Wang, "A symmetric image encryption algorithm based on mixed linear-nonlinear coupled map lattice," Information Sciences, vol. 273, pp. 329-351, 2014.

[23] X. Y. Wang and J. M. Song, "Synchronization of the fractional order hyperchaos Lorenz systems with activation feedback control," Communications in Nonlinear Science and Numerical Simulation, vol. 14, no. 8, pp. 3351-3357, 2009.

[24] F. C. Zhang, X. F. Liao, and G. Y. Zhang, "Qualitative behaviors of the continuous-time chaotic dynamical systems describing the interaction of waves in plasma," Nonlinear Dynamics, vol. 88, no. 3, pp. 1623-1629, 2017.

[25] F. C. Zhang, X. F. Liao, and G. Y. Zhang, "On the global boundedness of the Lü system," Applied Mathematics and Computation, vol. 284, pp. 332-339, 2016.

[26] F. C. Zhang, C. L. Mu, S. M. Zhou, and P. Zheng, "New results of the ultimate bound on the trajectories of the family of the Lorenz systems," Discrete \& Continuous Dynamical Systems - B, vol. 20, no. 4, pp. 1261-1276, 2015.

[27] F. C. Zhang, X. F. Liao, C. L. Mu, G. Y. Zhang, and Y. A. Chen, "On global boundedness of the Chen system," Discrete \& Continuous Dynamical Systems - B, vol. 22, no. 4, pp. 16731681, 2017.

[28] A. Pogromsky and H. Nijmeijer, "On estimates of the Hausdorff dimension of invariant compact sets," Nonlinearity, vol. 13, no. 3, pp. 927-945, 2000.

[29] F. C. Zhang and G. Y. Zhang, "Further results on ultimate bound on the trajectories of the Lorenz system," Qualitative Theory of Dynamical Systems, vol. 15, no. 1, pp. 221-235, 2016.

[30] F. C. Zhang, X. F. Liao, G. Y. Zhang, and C. L. Mu, "Dynamical analysis of the generalized Lorenz systems," Journal of Dynamical and Control Systems, vol. 23, no. 2, pp. 349-362, 2017.

[31] X. X. Liao, G. P. Zhou, Q. G. Yang, Y. L. Fu, and G. R. Chen, "Constructive proof of Lagrange stability and sufficientnecessary conditions of Lyapunov stability for Yang-Chen chaotic system," Applied Mathematics and Computation, vol. 309, pp. 205-221, 2017.

[32] F. C. Zhang, C. L. Mu, P. Zheng, D. Lin, and G. Y. Zhang, "The dynamical analysis of a new chaotic system and simulation," Mathematical Methods in the Applied Sciences, vol. 37, no. 12, pp. 1838-1846, 2014.

[33] C. Ma and X. Y. Wang, "Hopf bifurcation and topological horseshoe of a novel finance chaotic system," Communications in Nonlinear Science and Numerical Simulation, vol. 17, no. 2, pp. 721-730, 2012. 


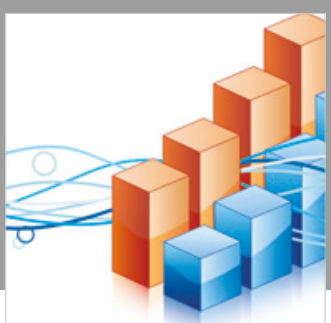

Advances in

Operations Research

\section{-n-m}
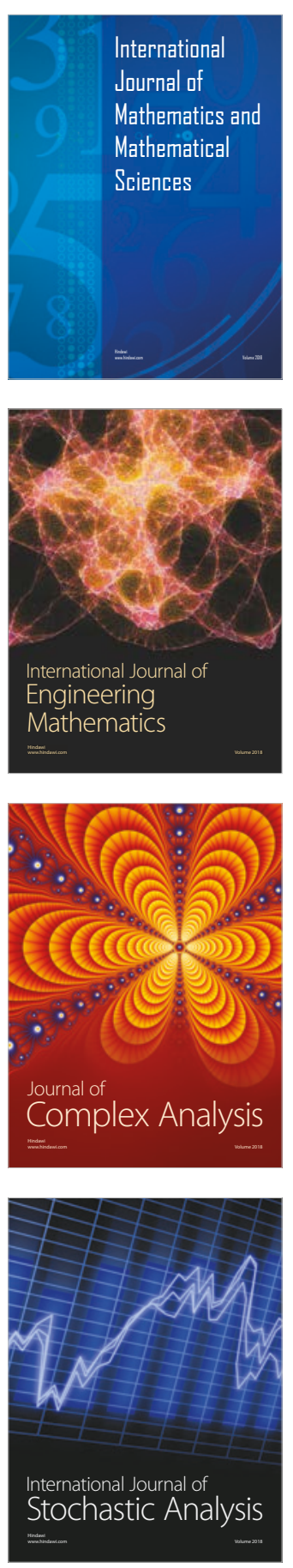
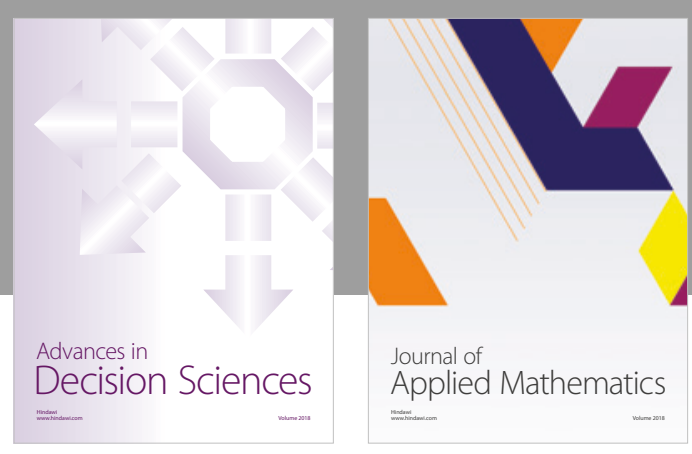

Journal of

Applied Mathematics
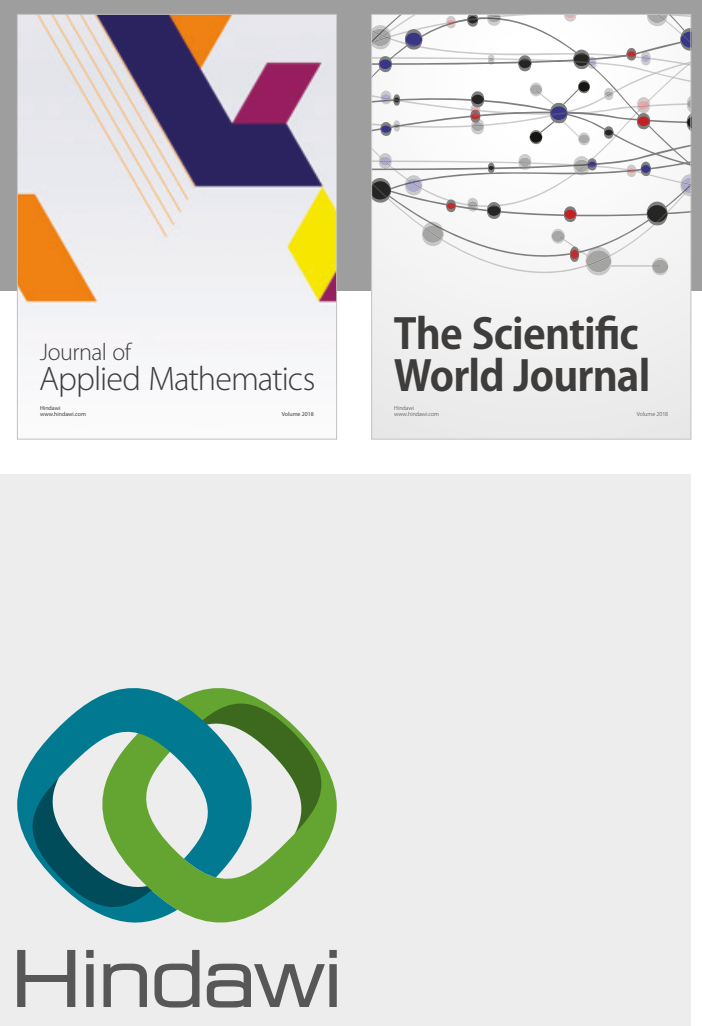

Submit your manuscripts at

www.hindawi.com

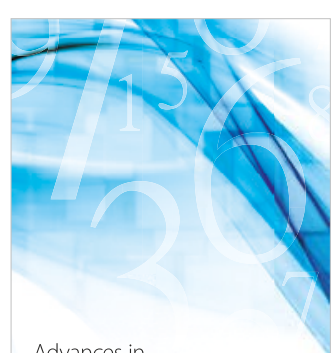

Advances in
Numerical Analysis
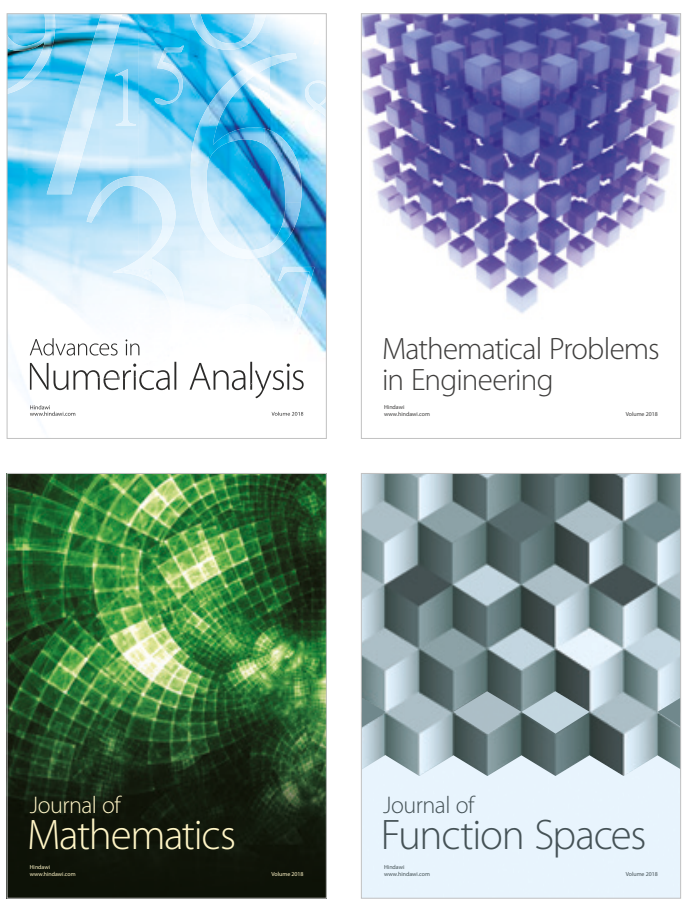

Mathematical Problems in Engineering

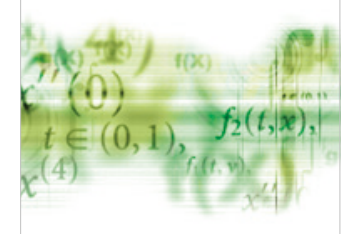

International Journal of

Differential Equations

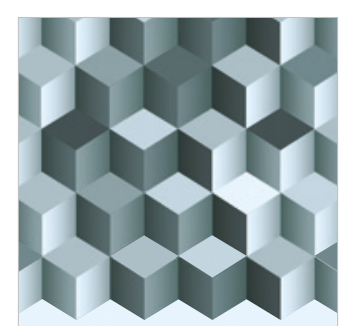

Journal of

Function Spaces
The Scientific

World Journal

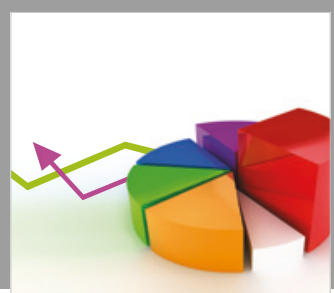

Journal of

Probability and Statistics
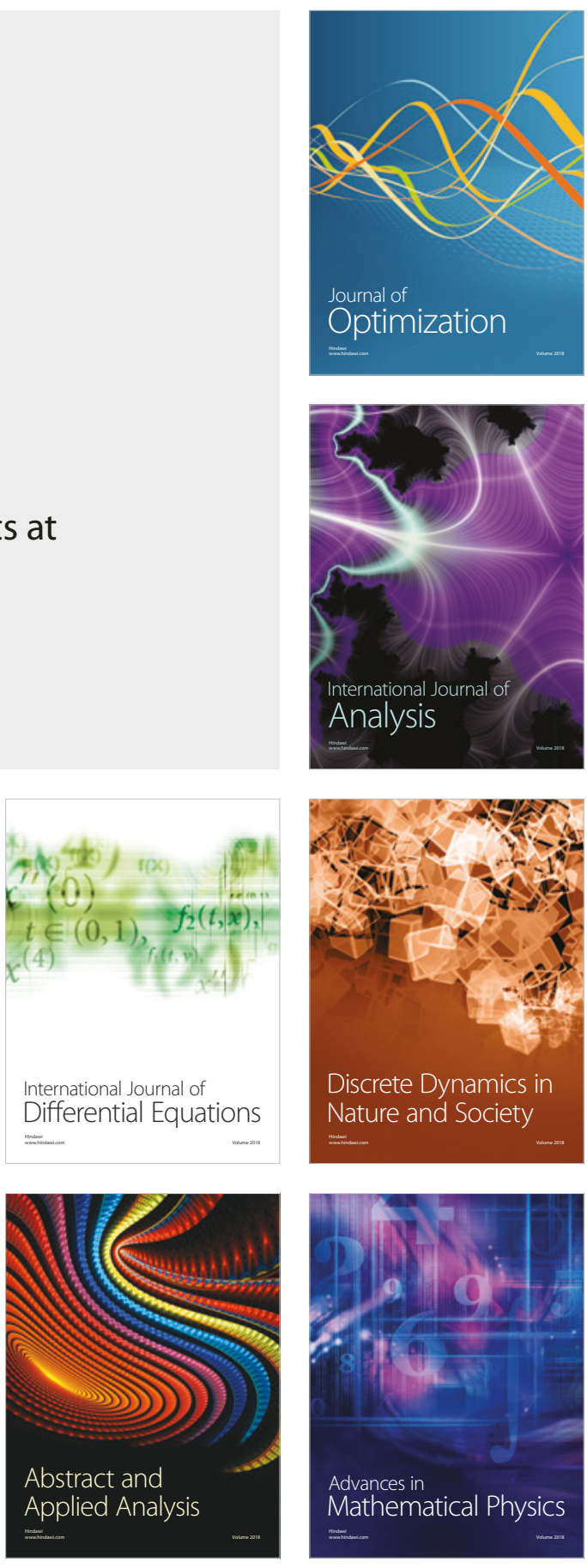